\title{
Eye Location Method based on gray projection with improved skin color segmentation
}

\author{
Feng Kai Ping \\ Guangdong University of Technology \\ GuangDong,GuangZhou,China \\ 461090628@qq.com
}

\author{
Pan Yang \\ Guangdong University of Technology \\ GuangDong,GuangZhou,China \\ 1019510674@qq.com
}

\begin{abstract}
The accuracy of eye location using gray projection method is dependent on the quality of the ambient light, the face posture and facial hair in front of the face. in order to solve the actual environment problem, this paper proposes a improved color segmentation and repeatedly reused gray projection which perform four times gray projection in face area.The experiments show this detection method can be used to avoid the disturb of actual environment and it can be up to 20 frames per second or more.This method can detected different human faces in different poses, the accuracy and efficiency of this algorithm has high robustness.
\end{abstract}

Keywords-gray Projection; eye location; skin color segmentation; otsu

\section{INTRODUCTION}

Eye location plays an important role in the current field of human-computer interaction. There are many ways to detect the human eye,such as reference[1][7] using template matching, has good robustness in normal posture and normal environment in eye location, but unable to adapt to the face region which is too small and tilted face. Reference[2][3][4][12] are using the gray projection method which is popular now. This method is product two histogram curve in the target image on the horizontal and vertical directions.Then, According the constraint of face morphology,we can the locate the human eye on a particular peak in the histogram curve.But this way is depend on the accuracy of the result in face detection.Such as reference[3] has good robustness in normal environment, however it will lose the robustness quickly when the environment become complex or the light become poor.reference[4] use the Image moments to detect the face area,but it only works in simple background.

Reference[5] propose a method of the combine between YUV and Otsu,but it can't be used to detect human eyes in video because it is too slow and it will perform a faulty detection when the background contains some objective which color is similar with human skin. So this paper is try to overcome the obstruction product by complex background,the surrounding interference in face area.and improve the speed of algorithm for the real time detection.

\section{GRAY PROJECTION ALGORITHM OPTIMIZATION DESIGN}

\section{A. The framework of eye location algorithm}

In order to get the correct face region, first, we should decrease the obstruction of background.Reference[6][10][11] have proved the skin color has great polymerizing in the $\mathrm{Cr}$ component of YCrCb color space.This thesis's method is based on reference[5] which is using the Otsu algorithm to search the best threshold. It will transform the RGB color space to $\mathrm{YCrCb}$ color space,then extract the $\mathrm{Cr}$ component when the camera send the graphic data to our computer. The second step is used Otsu algorithm to search the best threshold in Cr component, and this paper proposed add a compensation value to the threshold before produce a binarized image. After that, we are going to using Floodfill algorithm to eliminate the interference around the face area. Finally,this paper will perform a new gray projection method on the binarized image to locate the eyes. All the steps are showed in Fig.1.

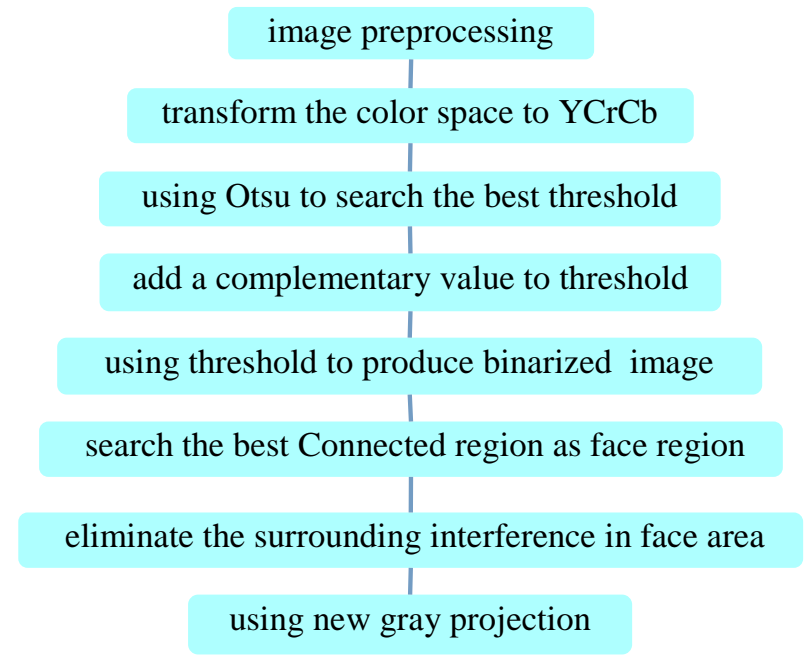

Figure.1 The framework of eye location algorithm 


\section{B. Add a compensation value to threshold}

When we got the threshold obtained by Otsu, we add a complementary value to the binary equation. The equation is shown as (1).

$$
f(x, y)= \begin{cases}255, & p(x, y)>T+\alpha \\ 0, & p(x, y)>T+\alpha\end{cases}
$$

The letter ' $\mathrm{T}$ ' is the threshold obtained by Otsu and the ' $a$ ' is the new parameter. When the complexity of background is changed,the parameter ' $\alpha$ ' often need to be set another figure.There is a lot of way to set the parameter ' $a$ '. This paper is use a way that move the face to fit the blue box. It means that let the connected region of face fit the box in binarized image.The effect of method is shown as Fig.2 and Fig.3.

\section{location}

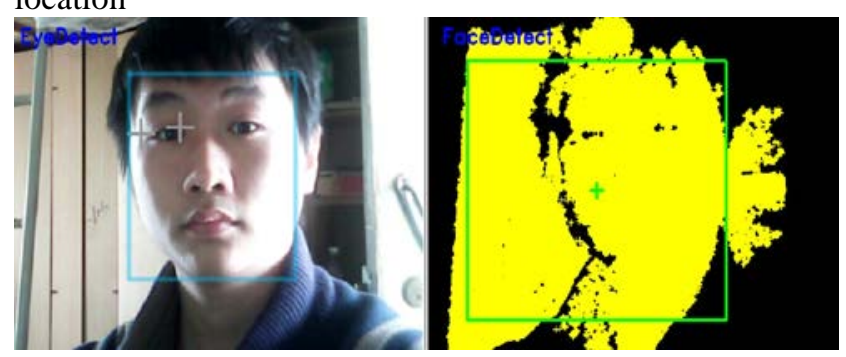

Figure.2 The result of face detection when $\alpha=0$

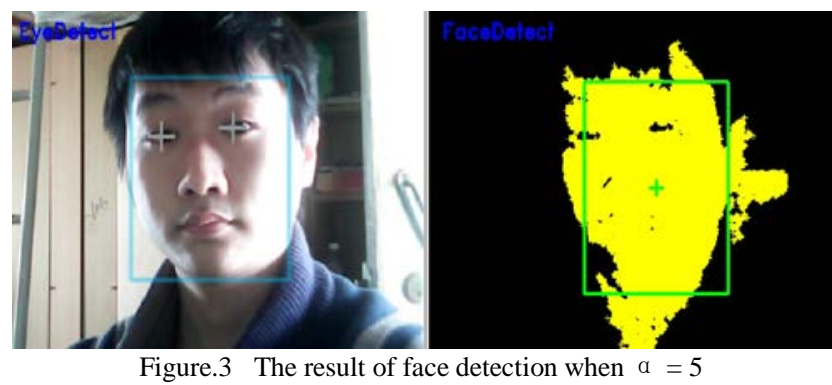

We can see that there is a wardrobe which Cr component is similar with human face and a side light on the right in Fig.2 and Fig.3. The Fig.2 show that the face detection will be failure when $a=0$, that means just depend on the Otsu threshold. After adjust the a to a suitable figure,face detecting will perform correctly.

\section{Eliminate the surrounding interference in face area}

After we obtain the binarized image by new threshold, the next step is using opening operation to eliminate the interference of noise[13] and search the best connected region as face region[4]. However, there is surrounding interference remain.This paper propose that using floodfill in the edge of face region.The target area which performed with floodfill can be set in 1/36 in face region.
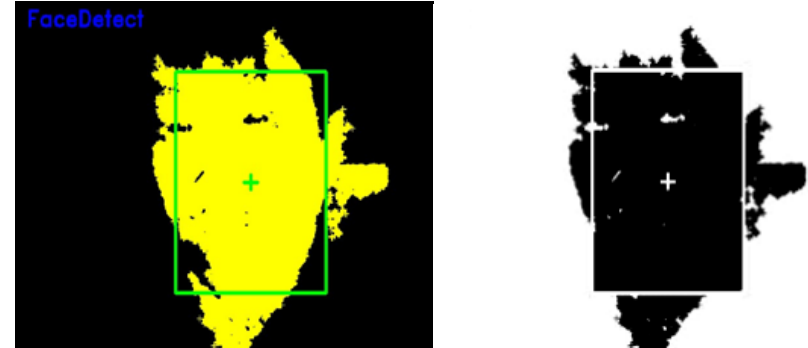

Figure.4 Using floodfill to eliminate the surrounding interference in face area

As is shown in Fig.4, the eyes region is not connected to the surrounding interference of the human region and The floodfill can eliminate the interference perfectly.

\section{Multiple gray projection}

When obtained the face region picture which is shown in the right of Fig.4, The new method, multiple gray projection can be used to locate the eyes. The process is shown in Fig.5 and the gray projection is show in Fig.6.

$$
\begin{aligned}
& \text { execute the vertical gray projection and select the max peak } \\
& \text { determine the max peak located left cheek or right cheek } \\
& \text { execute the vertical gray projection in another cheek and } \\
& \text { get another peak location } \\
& \text { execute the horizontal gray projection respectively between } \\
& \text { left cheek and right cheek to obtain the vertical coordinate } \\
& \text { combine the corresponding peak to create the eyes location } \\
& \text { Figure.5 The Framework of multiple gray projection }
\end{aligned}
$$

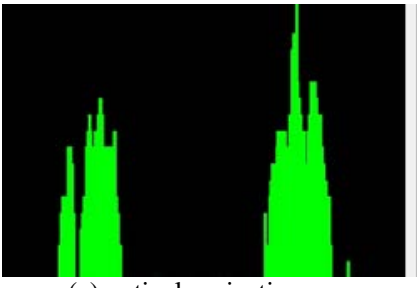

(a)vertical projection Figure.6

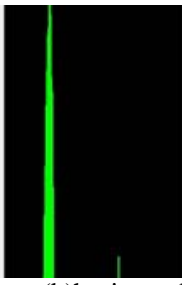

(b)horizontal projection

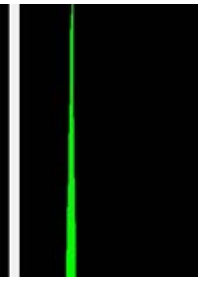

Gray projection

\section{ANALYSIS OF EXPERIMENTAL RESULTS}

This algorithm is compiled into MFC interface program by ViusalStudio2013. In this experiment, VidTIMIT face database and the camera are used to test the accuracy and efficiency of this algorithm . All the picture in VidTIMIT face database have a simple background and the face have different posture. we choose 50 pictures to perform the test. 
Then, we face to the camera to test the practicability in complicated background in 10 minutes.The background are student dormitory and laboratory.

\section{A. Intuitive results show}

As is shown in Fig.7, the experiments are located in student dormitory.There is no positive light and top light, but just the bright side.The background contain the wardrobe which Cr component are similar to human face.After added a complementary value to threshold and using floodfill to eliminate the surrounding interference, the eyes detection are performed correctly.

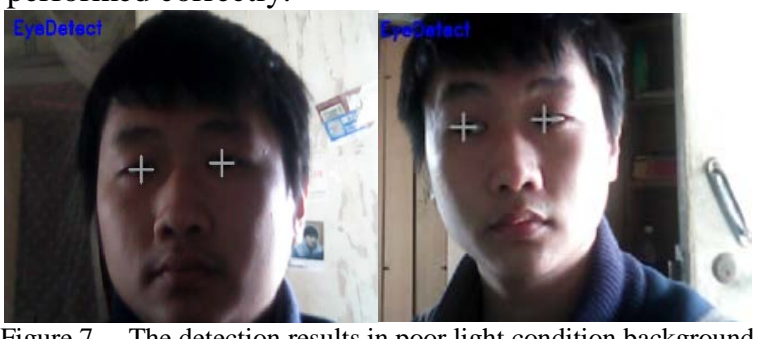

The next set are shown in Fig.8, The experiments are located in laboratory.This set are shown that the face tilted 45 degrees can be correctly located no matter the complicated background behind.

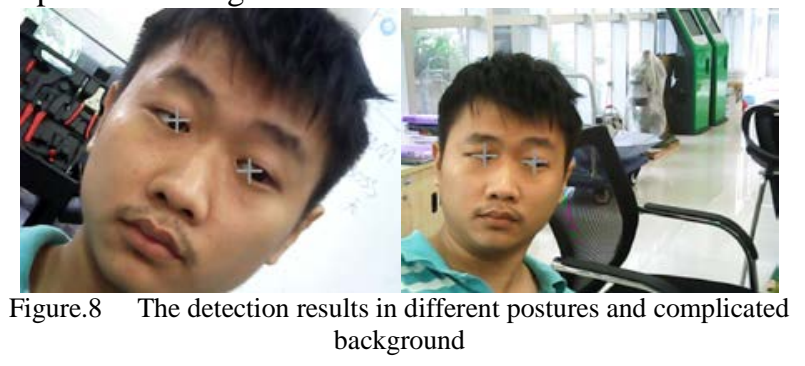

\section{B. The experimental data show}

The table.1 are compared the recently popular eyes location algorithm to this paper's method.

The second column in table. 1 are face database contained 50 pictures. All the picture selected form face database are simple background. The equation of accuracy ' $C$ ' are shown in (2) .

$$
\mathrm{C}=(\mathrm{T}-\mathrm{F}) / \mathrm{T}
$$

The ' $\mathrm{T}$ ' in (2) is the total quantity of samples which are contained 50 pictures in here. The ' $F$ ' is the fault location times.

The third column in table. 1 are faced to the camera 10 minute and change the posture at any time.The background are more complex than the image selected form VidTIMIT database. In this situation, The equation of accuracy ' $C$ ' which means the accuracy in camera model, and the ' $T$ ' means total frames in 10 minutes. At last, the ' $F$ ' means the fault location frames.

The reference[5] in table.1 is using YCbCr and Otsu to search human face but not add the complementary value to the threshold. It can perform a high accuracy in those picture with simple background, but when the background become complex, it will lose the accuracy quickly. Compared to this paper, the complementary value and eliminate the surrounding interference will increase the accuracy when face to the complex background. The reference[8] in table.1 is added Hough Transform, Although it can make a better performance when using face database to locate the eye, it can not reach a normal speed in video or faced to camera. However, this article using multiple gray projection can reach 20 FPS. The reference[9] is performing in changing illumination situation.its accuracy is low than this article. Therefore this article has some reference value to make a high speed performance and encounter complex background in eyes detecting.

\begin{tabular}{|l|l|l|l|}
\hline & $\begin{array}{l}\text { Accuracy } \\
\text { to face } \\
\text { database }\end{array}$ & $\begin{array}{l}\text { Accuracy in } \\
\text { camera model }\end{array}$ & Speed \\
\hline Reference[5] & $90.2 \%$ & $83.4 \%$ & $\approx 11 \mathrm{fps}$ \\
\hline Reference[8] & $93.2 \%$ & $85.4 \%$ & $\approx 7 \mathrm{fps}$ \\
\hline Reference[9] & $85 \%$ & $80.6 \%$ & $\approx 17 \mathrm{fps}$ \\
\hline This thesis & $92.6 \%$ & $87.8 \%$ & $\approx 20 \mathrm{fps}$ \\
\hline
\end{tabular}

Table.1 The comparison between this paper and other popular method.

\section{IN CONCLUSION}

As is shown in the experiment, the Otsu and YCrCb can not be made effective detection in some background with some object which Cr component is similar to human skin. In response to these problems, this paper presents a method that add a complementary value to the threshold and eliminate the surrounding interference in face area. Finally, using multiple gray projection to locate eyes quickly and accurately. However, add a complementary value to threshold is not enough in some case because the changed threshold may eliminate the detail,or increase the noise in face region. The next research is prepared to eliminate the increased noise and keep the detail in face.

\section{REFERENCES}

[1] SHU Mei, DONG Xiu-cheng, "Eye location based on skin color and template matching," Computer Engineering and Applications, 2009 , 45(2) : $237-239$.

[2] WANG Wen-cheng, "CHANG Fa-liang. A precise eye localization method based on region projection," Journal of Optoelectronics \& Laser, 2011, 22( 4):618 - 622.

[3] LIU Sha, CAI Can-hui, LIN Zhi-feng, "An Eye Fast Location Method Based On Complexion Detection," Computer Applications andSoftware, 2009, 10 ( 10) : $209-221$.

[4] WANG Kun, TIAN Xiang, WANG Wei-feng, "An improved eye location algorithm based on gray projection," Science Technology and Engineering, 2011-06-08.

[5] YANG Xiu-fang, QI Yin-cheng, LI Ting, YUAN Qing, "Eye Localization Optimization Method Based on YCb' Cr' Skin Color 
Feature and Otsu Algorithm,” Journal of Nanjing University of Posts and Telecommunications(Natural Science), Feb. 2014 No.1 Vol.34.

[6] FU Zhi-peng, WANG Feng, MA Xiao-qiang, "Eye Location Arithmetic Based on Gray Projection and Region Geometry Characters,” Computer Knowledge And Technology, Vol.4,No.7,December 2008, pp.1706-1708 .

[7] SUN Yan-feng, LIANG Yong-tao, SU Shi-qian, "Face Detection Based on skin color model and the center of gravity of the template," Vo1.32, No.5, Mav 2006.

[8] LI Ai-ping, WEI Jiang, HAO Si-si, "Eye location based on gray projection and improved Hough transform algorithm,” Vol.22, No.16, Aug.2014.

[9] SONG Hao-dong, LIN Xiao-zhu, "Eye Location under Varying Illumination," Journal of Beijing Institute of Graphic Communication, Vol. 22, No. 2, Apr. 2014.

[10] XU Qing, SHI Yue-xiang, XIE Wen-lan, "Method of face detection based on improved YUV color space," Computer Engineering and Applications, 2008, 44 (34): 158-162.

[11] YU Ming-hui, CHEN Wei, "Face Detection Algorithm of Double Color Model in Complex Illumination and Background. Journal of Jiangnan University(Natural Science Edition), Vol.13, No.5, Oct.2014.

[12] FENG Jian-qiang, LIU Wen-bo, YU Sheng - lin, "Eyes Location Based on Gray - level Integration Projection," Computer Simulation. Vol.4, 2005.

[13] YAN Li-ming, LI Yue, "Face Detection with Morphological Processing Based on OpenCV,” Industrial Control Computer. Vol.9, 2014. 